\title{
Découverte récente de Nyctalus leisleri en Corse
}

par R.M. LIBOIS

Laboratoire d'Ethologie U.Lg., quai Van Beneden, 22 B-4020 Liège et Station de Recherches sous-marines U.Lg. (STARESO), B.P. 33, F-20260 Calvi

Il y a peu, nous avons relaté la découverte d'un crâne de Myotis bechsteini à Olmi Capella (Haute Corse) dans un lot de pelotes de régurgitation de chouette effraie récolté en juillet 1980 (Libois et Vranken, 1981).

En novembre de la même année, nous retournions dans ce même village afin de compléter nos collections de crânes de mulots en vue d'une étude biométrique (voir Bordenave, 1981). A notre grande surprise, nous avons trouvé dans ces nouvelles pelotes un crâne et une mandibule gauche de Nyctalus leisleri Kiuhl, 1818. La composition de ce second lot est tout à fait semblable à celle du premier, tant qualitativement que quantitativement: nous y avons identifié 274 Apodemus sylvaticus, 58 Mus musculus, 17 Rattus rattus, 112 Crocidura suaveolens, 28 Suncus etruscus, 1 Barbastella barbastellus, 6 oiseaux, 3 batraciens et 4 insectes.

Dans la mesure où la noctule de Leisler est largement distribuée en Europe, sa présence en Corse n'a vraiment rien de surprenant d'autant plus que cette noctule est connue pour ses déplacements à longue distance (Krzanowski, 1960). Elle est d'ailleurs la seule chauve-souris des Açores (Corbet, 1970). La rareté de cet animal et le peu d'efforts consentis dans la recherche chiroptérologique en

Mammalia (1983), vol. 47 (3) : 425-426.

Corse expliquent qu'il n'y ait pas été détecté plus tôt. Quoi qu'il en soit, nos deux récentes trouvailles montrent la nécessité d'approfondir nos connaissances, fort fragmentaires encore, de la faune mammalogique corse tant l'intérêt biogéographique de l'île est remarquable.

\section{Données morphologiques (mensurations en $\mathrm{mm}$ ).}

Avant-bras : 41,40 env.

Crâne :

Long. I2-M3 : 6,20 env.

Long. M1-M3 : 4,05 env.

Long. P4-M3 : 4,65

Long. M3-M3 : 2,95

Largeur zygomatique : 10,15 env.

Larg. interorbitaire : 4,70

Larg. M3-M3 ext. : 6,90

Mandibule :

Long. I1- apophyse angulaire : 11,45

Long. I1- condyle : 11,30

N.B. - Comme les restes crâniens du vespertilion de Bechstein, ceux de la noctule de Leisler d'Olmi Capella sont conservés dans les collections du musée de zoologie de l'Université de Liège ( $\mathrm{n}^{\mathrm{os}}$ de registre respectifs : 13619 et 13620).

\section{Bibliographie.}

BoRDENAVE, D., 1981. - Statut taxonomique et biogéographie du Mulot, Apodemus sp., en Corse. Mém. Lic. Sc. zool. Univ. Liège, 64 pp. (non publié). CoRBET, G.B., 1970. Vagrant bats in Shetlands and the North Sea. J. Zool. Lond., 161 :
281-182.

KRZANowsKI, A., 1960. - Investigations of flights of Polish bats, mainly Myotis myotis (Borkhausen, 1797). Acta theriol., 4: 175-184.

Libois, R.M., et M. VRANKen, 1981. - Myotis bechsteini en Corse. Mammalia, 45 : 380-381. 